\title{
Impact of academic collaboration and quality of clinical orthopaedic research conducted in low- and middle-income countries
}

\author{
Hao-Hua Wu ${ }^{1, *}$, Max Liu ${ }^{1}$, Kushal R. Patel ${ }^{2}$, Wes Turner ${ }^{1}$, Lincoln Baltus ${ }^{1}$, Amber M. Caldwell ${ }^{1}$,
} Jesse C. Hahn ${ }^{1}$, Ralph Richard Coughlin ${ }^{1}$, Saam Morshed ${ }^{1}$, Theodore Miclau ${ }^{1}$, and David W. Shearer ${ }^{1}$

1 Department of Orthopaedic Surgery, Orthopaedic Trauma Institute, Institute for Global Orthopaedics and Traumatology, University of California, San Francisco, San Francisco, CA 94110, USA

2 Department of Orthopaedic Surgery, University of Illinois College of Medicine, Chicago, IL 60612, USA

Received 3 May 2016, Accepted 24 November 2016, Published online 30 January 2017

\begin{abstract}
Background: Little is known about the quality of orthopaedic investigations conducted in low- and middle-income countries (LMICs). Academic collaboration is one model to build research capacity and improve research quality. Our study aimed to determine (1) the quality of clinical orthopaedic research conducted in LMICs, (2) the World Bank Regions and LMICs that publish the highest quality studies, (3) the pattern of collaboration among investigators and (4) whether academic collaboration between LMIC and non-LMIC investigators is associated with studies that have higher levels of evidence.

Methods: Orthopaedic studies from 2004 to 2014 conducted in LMICs were extracted from multiple electronic databases. The World Bank Region, level of evidence and author country-affiliation were recorded. Collaboration was defined as a study that included an LMIC with non-LMIC investigator.

Results: There were 958 studies that met inclusion criteria of 22,714 searched. Ninety-seven (10.1\%) of included studies achieved Level 1 or 2 evidence, but case series (52.3\%) were the most common. Collaboration occurred in $14.4 \%$ of studies and the vast majority of these $(88.4 \%)$ were among academic institutions. Collaborative studies were more likely to be Level 1 or $2(20.3 \%$ vs. $8.4 \%, p<0.01)$, prospective $(34.8 \%$ vs. $22.9 \% p=0.04)$ and controlled $(29.7 \%$ vs. $14.4 \%, p<0.01)$ compared to non-collaborative studies.

Conclusions: Although orthopaedic studies in LMICs rarely reach Level 1 or 2 evidence, studies published through academic collaboration between LMIC and non-LMIC investigators are associated with higher levels of evidence and more prospective, controlled designs.
\end{abstract}

Key words: Orthopaedics, Global surgery, Low- and middle-income Countries, Clinical research, Academic collaboration, Levels of evidence, Research capacity.

\section{Introduction}

There is increasing recognition of the importance of musculoskeletal disease as a global health issue, particularly as it relates to injury. An estimated 1.2 million deaths and 50 million nonfatal injuries occur each year due to road traffic accidents alone [1], with traumatic injuries contributing to more global disability than human immunodeficiency virus (HIV), tuberculosis and malaria combined $[2,3]$. The burden of injury is greatest in low- and middle-income countries (LMICs), where resource-limited orthopaedic surgeons face a daunting volume of musculoskeletal disease [2, 4]. Efforts

\footnotetext{
*Corresponding author: haohuawu@gmail.com
}

to address this disparity in care have manifested through surgical missions, donated implants and educational programmes $[4,5]$. Less, however, is known about the impact of clinical research $[4,5]$. Recent literature suggests that clinical orthopaedic research conducted in LMICs may help to answer relevant clinical questions and shape public health policy [4]. Although a growing body of research exists to inform treatment for orthopaedic disease in high-income countries (HICs), generalizing results to LMICs is difficult due to delays in treatment, differences in training and availability of equipment $[6,7]$. Thus, it would be ideal for orthopaedic surgeons practicing in LMICs to base their treatment on high-quality research conducted in a similar setting [4]. 
Table 1. Countries with low-income or lower-middle-income economies as defined by The World Bank. Table adapted from http://data.worldbank.org/about/country-and-lending-groups.

\begin{tabular}{|c|c|c|}
\hline \multicolumn{3}{|c|}{ Low-income economies ( $\$ 1045$ or less) $[n=31]$} \\
\hline Afghanistan & Gambia, The & Niger \\
\hline Benin & Guinea & Rwanda \\
\hline Burkina Faso & Guinea-Bisau & Sierra Leone \\
\hline Burundi & Haiti & Somalia \\
\hline Cambodia & Korea, Dem Rep. & South Sudan \\
\hline $\begin{array}{l}\text { Central African } \\
\text { Republic }\end{array}$ & Liberia & Tanzania \\
\hline Chad & Madagascar & Togo (Sub-Saharan) \\
\hline Comoros & Malawi & Uganda \\
\hline Congo, Dem. Rep & Mali & Zimbabwe \\
\hline Eritrea & Mozambique & \\
\hline Ethiopia & Nepal & \\
\hline \multicolumn{3}{|c|}{ Lower-middle-income economies ( $\$ 1046$ to $\$ 4125)[n=51]$} \\
\hline Armenia & Indonesia & Samoa \\
\hline Bangladesh & Kenya & $\begin{array}{l}\text { São Tomé and } \\
\text { Principe }\end{array}$ \\
\hline Bhutan & Kiribati & Senegal \\
\hline Bolivia & Kosovo & Solomon Islands \\
\hline Cabo Verde & Kyrgyz Republic & Sri Lanka \\
\hline Cameroon & Lao PDR & Sudan \\
\hline Congo, Rep. & Lesotho & Swaziland \\
\hline Côte d'Ivoire & Mauritania & Syrian Arab Republic \\
\hline Djibouti & $\begin{array}{l}\text { Micronesia, } \\
\text { Fed. Sts. }\end{array}$ & Tajikistan \\
\hline Egypt, Arab Rep. & Mongolia & Timor-Leste \\
\hline El Salvador & $\begin{array}{l}\text { Morocco (North } \\
\text { Africa) }\end{array}$ & Ukraine \\
\hline Georgia & Myanmar & $\begin{array}{l}\text { Uzbekistan (Central } \\
\text { Asia) }\end{array}$ \\
\hline Ghana & Nicaragua & Vanuatu \\
\hline Guatemala & $\begin{array}{l}\text { Nigeria } \\
\text { (West Africa) }\end{array}$ & Vietnam \\
\hline Guyana & $\begin{array}{l}\text { Pakistan } \\
\quad \text { (South Asia) }\end{array}$ & West Bank and Gaza \\
\hline Honduras & Papua New Guinea & Yemen, Rep. \\
\hline India & Philippines & Zambia \\
\hline
\end{tabular}

To date, the quality of clinical research conducted in LMICs has not been assessed. Orthopaedic surgeons weigh a study's level of evidence (LOE) before determining how the results apply clinical practice [8-10], as Level 1 or 2 studies can potentially reveal important therapeutic, diagnostic, prognostic and economic outcomes $[11,12]$. Given the importance of study quality in shaping treatment protocol, the state of orthopaedic research in developing countries requires comprehensive review. Some have hypothesized that collaboration between investigators from LMICs and non-LMICs (e.g. upper-middle and high-income countries) can improve study quality $[4,13]$. While there is growing evidence that investigators from LMICs strive to answer clinically relevant questions through research, they may lack resources, training and protected time away from clinical responsibilities [14]. However, collaboration with academically affiliated non-LMIC authors may help local investigators overcome these barriers through funding support, research education and division of labour [4]. Thus, investigations are needed to evaluate how international academic partnership can improve study quality.
Our study aimed to determine (1) the quality of clinical orthopaedic research conducted in LMICs, (2) the World Bank Regions and LMICs that publish the highest quality studies, (3) the pattern of collaboration among investigators and (4) whether academic collaboration between LMIC and nonLMIC investigators is associated with studies that have higher levels of evidence.

\section{Material and methods}

We conducted a scoping review using the Arksey and O'Malley framework with modifications from Levac et al. and Daudt et al. [15-17]. Five authors sought and assessed orthopaedic studies conducted in LMICs that were published between June 2004 and June 2014. The LMICs were defined as any country listed as a "Low-income" or "Lower-middleincome" economy according to the 2014 World Bank classification [18]. Eighty-two countries met our study's definition of LMIC (Table 1). The studies were identified by comprehensive textword and MeSH-based electronic searches of PubMed/ MEDLINE, EMBASE and Cochrane library that was developed with assistance from a research librarian. Our search strategy combined terms for orthopaedic surgery, LMICs, musculoskeletal injury, musculoskeletal anatomy and human studies to be as inclusive as possible (Appendix).

Full-text articles were assessed for eligibility in the order of title, abstract and manuscript. Included studies (1) deal primarily with a low- or lower-middle-income country (LMIC), (2) pertain to orthopaedic surgery, (3) enrol humans and (4) were original peer-reviewed publications. Studies that reported three cases or fewer, were non-English and pertained to a highincome country (HIC) at war, animals, biomechanics or laboratory values were excluded.

Each included study was read in its entirety and a REDCap survey for data extraction was created that identified the study location, author's academic affiliation and study quality. Study location and author affiliations were categorized into eight World Bank Regions: East Asia and Pacific, Europe and Central Asia, Latin America and the Caribbean, Middle East and North Africa, Latin America and the Caribbean, Middle East and North Africa, North America, South Asia (Table 2). Collaboration was determined by the investigator countryaffiliation and defined as LMIC-only, Multicentre (LMIC with LMIC investigator), Collaborative (LMIC with non-LMIC investigator) and non-LMIC (e.g. upper-middle or high-income country investigators) only. Academic partnerships were defined as a study where the authors from both LMICs and non-LMICs were affiliated with an academic institution.

Finally, the study quality was assessed by the levels of evidence (LOE), presence of control group, prospective or retrospective design, type of study and presence of randomization. Due to the heterogeneity of study design in the global orthopaedic literature, which includes qualitative and epidemiologic studies that cannot be categorized with the LOE scale, no other quality assessment tools were utilized. To determine the level of evidence of each study, we used the 2015 scale adopted by the Journal of Bone and Joint Surgery and derived from recommendations given by the Centre for Evidence-Based 
Table 2. Countries by World Bank Regions, adapted from http://data.worldbank.org/about/country-and-lending-groups.

\begin{tabular}{|c|c|c|}
\hline \multicolumn{3}{|c|}{ East Asia and Pacific, $n=37$} \\
\hline American Samoa & Korea, Rep. & Philippines \\
\hline Australia & Lao PDR & Samoa \\
\hline Brunei Darussalam & Macao SAR, China & Singapore \\
\hline Cambodia & Malaysia & Solomon Islands \\
\hline China & Marshall Islands & Taiwan, China \\
\hline Fiji & \multicolumn{2}{|c|}{ Micronesia, Fed. Sts.Thailand } \\
\hline French Polynesia & Mongolia & Timor-Leste \\
\hline Guam & Myanmar & Tonga \\
\hline $\begin{array}{l}\text { Hong Kong SAR, } \\
\text { China }\end{array}$ & New Caledonia & Tuvalu \\
\hline Indonesia & New Zealand & Vanuatu \\
\hline Japan & $\begin{array}{l}\text { Northern Mariana } \\
\text { Islands }\end{array}$ & Vietnam \\
\hline Kiribati & Palau & \\
\hline Korea, Dem. Rep. & Papua New Guinea & \\
\hline \multicolumn{3}{|c|}{ Europe and Central Asia, $n=57$} \\
\hline Albania & Germany & Netherlands \\
\hline Andorra & Greece & Norway \\
\hline Armenia & Greenland & Poland \\
\hline Austria & Hungary & Portugal \\
\hline Azerbaijan & Iceland & Romania \\
\hline Belarus & Ireland & Russian Federation \\
\hline Belgium & Isle of Man & San Marino \\
\hline $\begin{array}{l}\text { Bosnia and } \\
\text { Herzegovina }\end{array}$ & Italy & Serbia \\
\hline Bulgaria & Kazakhstan & Slovak Republic \\
\hline Channel Islands & Kosovo & Slovenia \\
\hline Croatia & Kyrgyz Republic & Spain \\
\hline Cyprus & Latvia & Sweden \\
\hline Czech Republic & Liechtenstein & Switzerland \\
\hline Denmark & Lithuania & Tajikistan \\
\hline Estonia & Luxembourg & Turkey \\
\hline Faeroe Islands & Macedonia, FYR & Turkmenistan \\
\hline Finland & Moldova & Ukraine \\
\hline France & Monaco & United Kingdom \\
\hline Georgia & Montenegro & Uzbekistan \\
\hline \multicolumn{3}{|c|}{ Latin America and the Caribbean, $n=41$} \\
\hline $\begin{array}{l}\text { Antigua and } \\
\text { Barbuda }\end{array}$ & Dominica & Peru \\
\hline Argentina & \multicolumn{2}{|c|}{ Dominican Republic Puerto Rico } \\
\hline Aruba & Ecuador & Sint Maarten (Dutch part) \\
\hline Bahamas, The & El Salvador & St. Kitts and Nevis \\
\hline Barbados & Grenada & St. Lucia \\
\hline Belize & Guatemala & St. Martin (French part) \\
\hline Bolivia & Guyana & $\begin{array}{l}\text { St. Vincent and the } \\
\text { Grenadines }\end{array}$ \\
\hline Brazil & Haiti & Suriname \\
\hline Cayman Islands & Honduras & Trinidad and Tobago \\
\hline Chile & Jamaica & Turks and Caicos Islands \\
\hline Colombia & Mexico & Uruguay \\
\hline Costa Rica & Nicaragua & Venezuela, RB \\
\hline Cuba & Panama & Virgin Islands (U.S.) \\
\hline Curacao & Paraguay & \\
\hline \multicolumn{3}{|c|}{ Middle East and North Africa, $n=21$} \\
\hline Algeria & Jordan & Qatar \\
\hline Bahrain & Kuwait & Saudi Arabia \\
\hline Djibouti & Lebanon & Syrian Arab Republic \\
\hline Egypt, Arab Rep. & Libya & Tunisia \\
\hline Iran, Islamic Rep. & Malta & United Arab Emirates \\
\hline Iraq & Morocco & West Bank and Gaza \\
\hline
\end{tabular}

\begin{tabular}{|c|c|c|}
\hline \multirow{2}{*}{\multicolumn{3}{|c|}{$\begin{array}{l}\text { Israel Oman } \\
\text { North America, } n=3\end{array}$}} \\
\hline & & \\
\hline Bermuda & Canada & United States \\
\hline \multicolumn{3}{|l|}{ South Asia, $n=8$} \\
\hline Afghanistan & India & Pakistan \\
\hline Bangladesh & Maldives & Sri Lanka \\
\hline Bhutan & Nepal & \\
\hline \multicolumn{3}{|c|}{ Sub-Saharan Africa, $n=48$} \\
\hline Angola & Gabon & Nigeria \\
\hline Benin & Gambia, The & Rwanda \\
\hline Botswana & Ghana & São Tomé and Principe \\
\hline Burkina Faso & Guinea & Senegal \\
\hline Burundi & Guinea-Bissau & Seychelles \\
\hline Cabo Verde & Kenya & Sierra Leone \\
\hline Cameroon & Lesotho & Somalia \\
\hline $\begin{array}{l}\text { Central African } \\
\text { Republic }\end{array}$ & Liberia & South Africa \\
\hline Chad & Madagascar & South Sudan \\
\hline Comoros & Malawi & Sudan \\
\hline Congo, Dem. Rep. & Mali & Swaziland \\
\hline Congo, Rep & Mauritania & Tanzania \\
\hline Côte d'Ivoire & Mauritius & Togo \\
\hline Equatorial Guinea & Mozambique & Uganda \\
\hline Eritrea & Namibia & Zambia \\
\hline Ethiopia & Niger & Zimbabwe \\
\hline
\end{tabular}

Medicine in Oxford, United Kingdom [19, 20]. The LOE scale divides studies into four categories: Diagnostic, Prognostic, Therapeutic, and Economic and Decision Analyses. Each category can then be subdivided into Level 1-5 evidence, with each level having its own definition [19]. Level 1 and 2 studies were considered high levels of evidence, while those of Level 3, 4 and 5 were considered low levels of evidence. Studies that had no levels of evidence, such as epidemiologic or qualitative studies, were also noted. LOE was used as the primary indicator of study quality due to its ubiquitous use as a validated measure of study strength [9, 11, 21-23]. In addition, studies that do include control groups, collect data prospectively and randomize interventions have been shown to produce higher quality evidence than studies that do not $[8,10]$.

\section{Data analysis}

Descriptive statistics were used to summarize all data. Discrete variables were summarized as counts or proportions, and skewed continuous variables were reported as medians with interquartile ranges (IQRs). Chi-square analysis was used to determine the association between nominal variables. Significance was set at $p<0.05$.

\section{Results}

Out of 22,714 unique articles assessed, 958 met all inclusion criteria (Figure 1). Over the past decade, 265 (27.6\%) of clinical orthopaedic research studies conducted in LMICs were epidemiologic or qualitative and had no LOE. Of the clinical studies that could be assessed with levels of evidence, only $97(10.1 \%)$ were Level 1 or 2 . Studies were most commonly designed as prospective or retrospective case series 


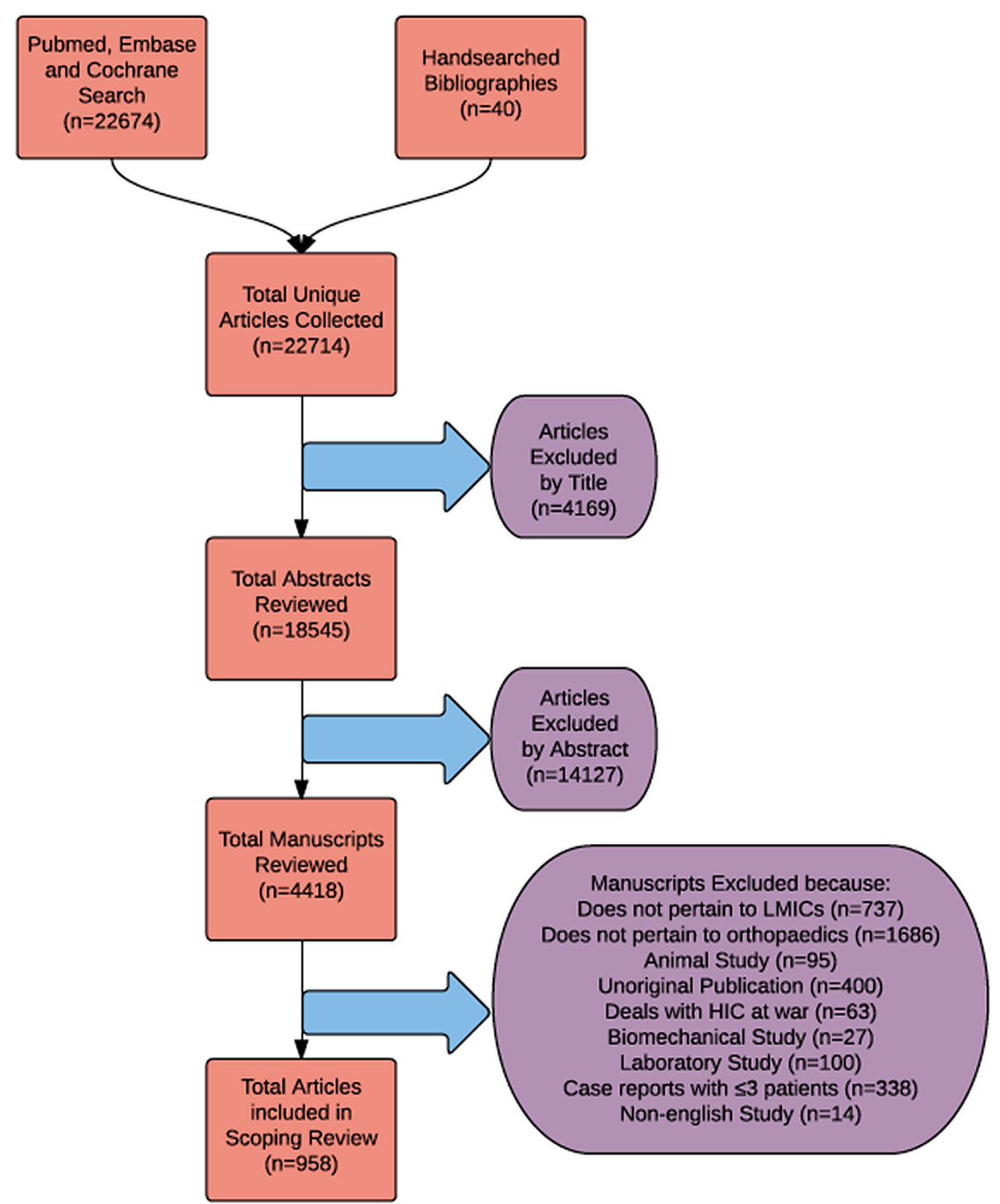

Figure 1. Flowchart shows included and excluded studies from search to data extraction.

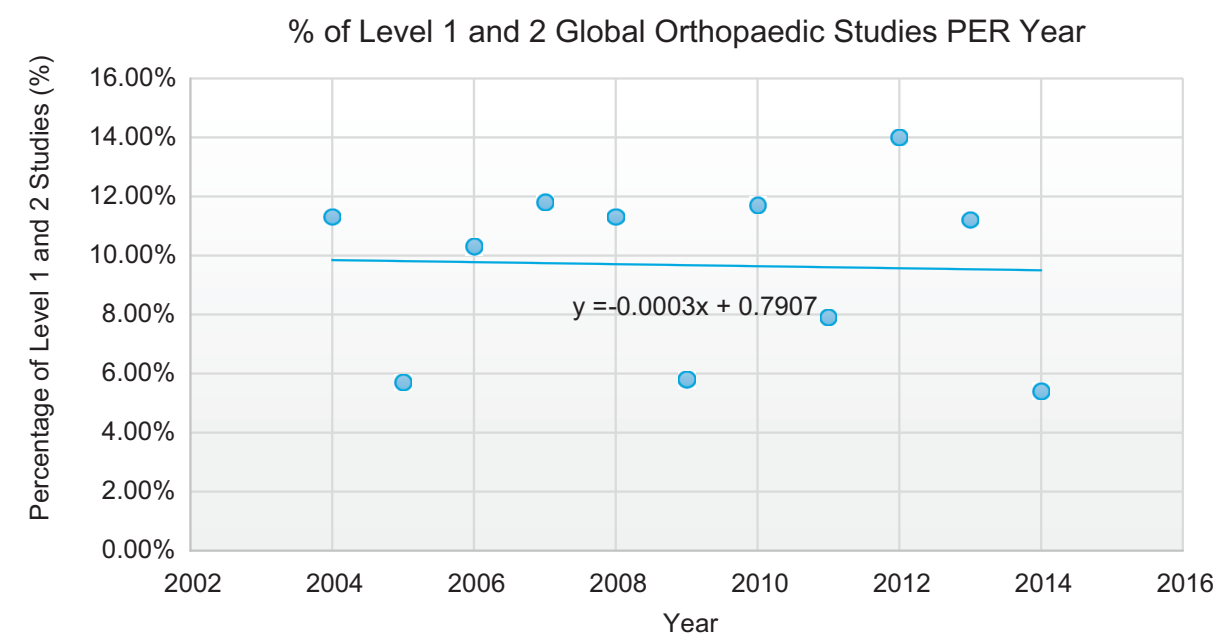

Figure 2. Percentage of Level 1 and 2 global orthopaedic studies conducted in low- and middle-income countries per year from June 2004 to June 2014. 


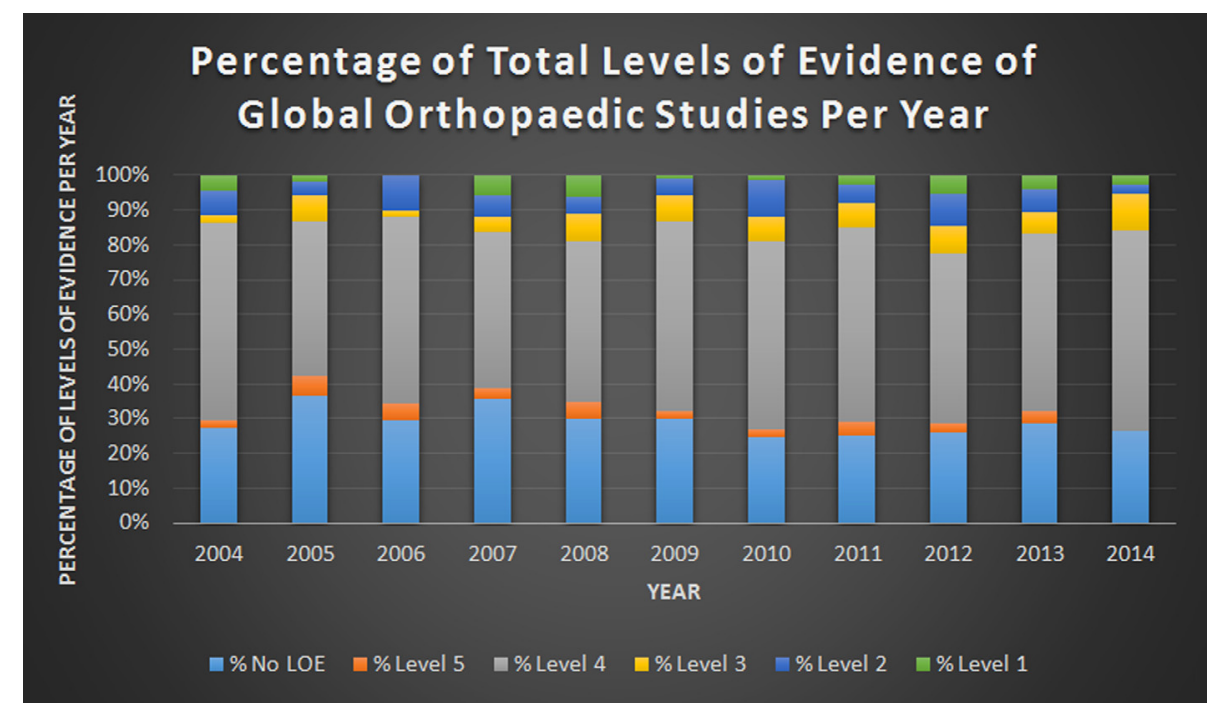

Figure 3. Percentage of total levels of evidence of global orthopaedic studies conducted in low- and middle-income countries per year from June 2004 June 2014.

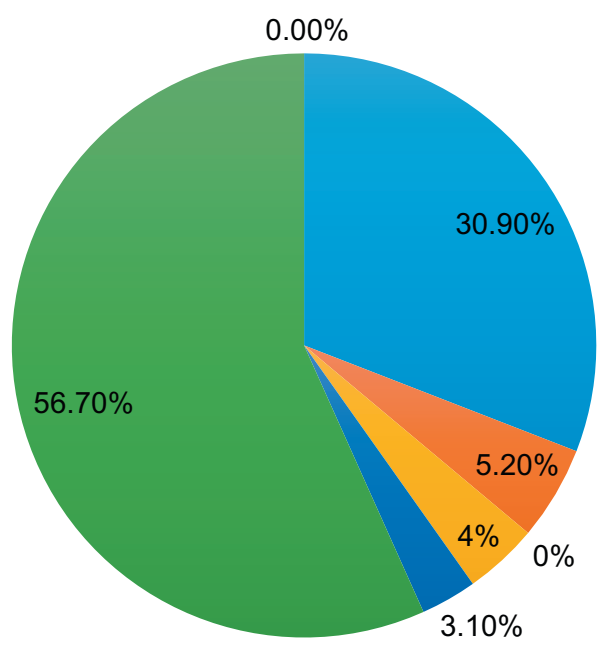

\author{
- Sub-Saharan Africa \\ East Asia and Pacific \\ - Europe and Central Asia \\ - Latin America and Caribbean \\ - Middle East and North Africa \\ - South Asia \\ - North America
}

Figure 4. Percentage of high-quality studies (e.g. Levels 1 and 2) published by World Bank Region from 2004 to 2014.

(501 [52.3\%]). In addition, only $158(16.5 \%)$ of all studies had a control group and only $24(2.5 \%)$ underwent randomization. Overall, the majority of data from these studies (75.4\%) were collected retrospectively. From 2004 to 2014, the median percentage of global orthopaedic studies published per year with a high LOE (e.g. Level 1 and 2 studies) was 11.2\% (IQR: 5.811.7). The percentage of high-quality global orthopaedics being conducted in LMICs annually has been stagnant over time (Figure 2), even though the number of LMIC orthopaedic studies has steadily increased over the same time period. A chisquare contingency table shows no statistically significant difference in the percentage of Level 1 and 2 studies published over the past decade $(p=0.4)$ (Figure 3 ). Of note, out of 503 therapeutic studies, 374 studies recommended the use of tested treatment or intervention, even though most of the studies $394(78.3 \%)$ were Levels 4 and 5 with respect to evidence.

Most studies took place in South Asia (48.4\%) and SubSaharan Africa (32.6\%). Of the 97 studies that were Level 1 or 2 evidence, $55(56.7 \%)$ took place in South Asia and
$30(30.9 \%)$ took place in Sub-Saharan Africa (Figure 4). Pakistan (25), India (19), Nigeria (14) and Nepal (9) were the LMICs with the most Level 1 and 2 studies published in the past decade (Table 3). Out of the 82 countries classified as LMICs by the World Bank, only $23(28.0 \%)$ had published a Level 1 or 2 orthopaedic study in the past decade. LMICs from the Middle East and North Africa (3.1\%), Latin America and Caribbean (4\%) and East Asia and Pacific (5.2\%) produced the lowest percentage of Level 1 and 2 studies since 2004.

Over the past decade, $138(14.4 \%)$ of global orthopaedic studies have been Collaborative, 30 (3.1\%) have been Multicentre, $85(8.9 \%)$ have only included authors from non-LMICs and $705(73.6 \%)$ have only included authors from LMICs. The majority $(75.8 \%)$ of them took place in academically affiliated institutions. In addition, the majority of non-LMIC authors practised either in Europe and Central Asia (44\%) or in North America (42.8\%) (Figure 5).

Out of the 138 studies where LMIC and non-LMIC authors worked together, $122(88.4 \%)$ of them represented academic 
Table 3. Study setting region, Level 1 and 2 studies by region and countries that have published Level 1 and 2 studies.

\begin{tabular}{|c|c|}
\hline Variables & Study characteristics $(n=958)$ \\
\hline \multicolumn{2}{|l|}{ Study setting region } \\
\hline Sub-Saharan Africa $(n[\%])$ & $312[32.6 \%]$ \\
\hline Level 1 or 2 studies $(n[\%])$ & $30[30.9 \%]$ \\
\hline $\begin{array}{l}\text { Number of Level } 1 \text { or } 2 \text { orthopaedic studies } \\
\text { published by country since } 2004\end{array}$ & $\begin{array}{l}\text { Nigeria (14), Malawi (5), Sudan (3), Cameroon (2), Kenya (2) } \\
\text { Ethiopia (1), Sierra Leone (1), Tanzania (1), Uganda (1) }\end{array}$ \\
\hline East Asia and Pacific $(n[\%])$ & $53[5.5 \%]$ \\
\hline Level 1 or 2 studies $(n[\%])$ & $5[5.2 \%]$ \\
\hline $\begin{array}{l}\text { Number of Level } 1 \text { or } 2 \text { orthopaedic studies } \\
\text { published by country since } 2004\end{array}$ & $\begin{array}{l}\text { Cambodia (1), Mongolia (1), Myanmar (1), } \\
\text { Philippines (1), Vietnam (1) }\end{array}$ \\
\hline Europe and Central Asia $(n[\%])$ & $17[1.8 \%]$ \\
\hline Level 1 or 2 studies $(n[\%])$ & $0[0 \%]$ \\
\hline Latin America and Caribbean ( $n[\%])$ & $30[3.1 \%]$ \\
\hline Level 1 or 2 studies $(n[\%])$ & $4[4.1 \%]$ \\
\hline $\begin{array}{l}\text { Number of Level } 1 \text { or } 2 \text { orthopaedic studies } \\
\text { published by country since } 2004\end{array}$ & Haiti (2), Guatemala (1), Guyana (1) \\
\hline Middle East and North Africa ( $n[\%])$ & $82[8.6 \%]$ \\
\hline Level 1 or 2 studies $(n[\%])$ & $3[3.1]$ \\
\hline $\begin{array}{l}\text { Number of Level } 1 \text { or } 2 \text { orthopaedic studies } \\
\text { published by country since } 2004\end{array}$ & Egypt (3) \\
\hline South Asia $(n[\%])$ & $464[48.4 \%]$ \\
\hline Level 1 or 2 studies $(n[\%])$ & $55[56.7 \%]$ \\
\hline $\begin{array}{l}\text { Number of Level } 1 \text { or } 2 \text { orthopaedic studies } \\
\text { published by country since } 2004\end{array}$ & $\begin{array}{l}\text { Pakistan (25), India (19), Nepal (9), Bangladesh (2), } \\
\text { Afghanistan (1) }\end{array}$ \\
\hline
\end{tabular}

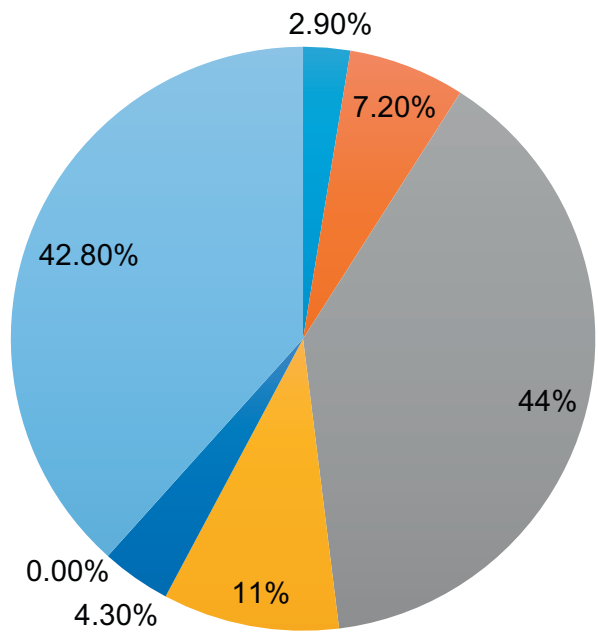

- Sub-Saharan Africa

- East Asia and Pacific

- Europe and Central Asia

- Latin America and Caribbean

- Middle East and North Africa

- South Asia

north America

Figure 5. Percentage of non-LMIC collaborative authors by World Bank Region from 2004 to 2014.

partnerships. Collaborative studies were more likely to be Level 1 or $2(20.3 \%$ vs. $8.4 \%, p<0.01)$, prospective $(34.8 \%$ vs. $22.9 \% p=0.04)$ and controlled $(29.7 \%$ vs. $14.4 \%$, $p<0.01$ ) compared to non-collaborative studies (Table 4). There was no difference in randomization between Collaborative and non-collaborative studies $(p=0.53)$.

\section{Discussion}

While the importance of evidence-based clinical practice is increasingly recognized in high-income countries, there is an enormous gap in the volume and quality of musculoskeletal research relevant to clinicians and patients in LMICs. Our study shows that the majority of clinical orthopaedic researches published in LMICs are designed as case series, and these studies rarely attain high levels of evidence, a phenomenon that has persisted over the past decade. Notably, there appears to be no significant difference between the proportion of Level 1 and 2 studies published in 2004 and the years leading up to 2014. Studies with low levels of evidence cannot accurately determine the treatment outcome and may be harmful if misinterpreted [11]. Our data shows that while the majority of therapeutic studies conducted in LMICs recommended the use of the tested intervention, they were mostly designed as case series. Given the importance of research in the effort to treat global orthopaedic disease, efforts to enable local researchers to produce high-quality studies are warranted. 
Table 4. Characteristics of collaborative global orthopaedic studies from 2004 to 2014.

\begin{tabular}{lc}
\hline \multicolumn{1}{c}{ Variables } & $\begin{array}{c}\text { Study characteristics } \\
(n=138)\end{array}$ \\
\hline $\begin{array}{l}\text { Region of collaborating site by WHO } \\
\text { classification }\end{array}$ & \\
Sub-Saharan Africa $(n[\%])$ & $4[2.9 \%]$ \\
North America $(n[\%])$ & $59[42.8 \%]$ \\
East Asia and Pacific $(n[\%])$ & $10[7.2 \%]$ \\
Europe and Central Asia $(n[\%])$ & $60[43.5 \%]$ \\
Middle East and North Africa $(n[\%])$ & $6[4.3 \%]$ \\
Latin America and Caribbean $(n[\%])$ & $15[10.9 \%]$ \\
South Asia $(n[\%])$ & $0[0 \%]$ \\
Quality of collaborative studies & \\
Level 1 and 2 studies $(n[\%])$ & $28(20.3 \%)$ \\
Level 3, 4, 5 studies $(n[\%])$ & $57(41.3 \%)$ \\
No Level of Evidence $(n[\%])$ & $53(38.4 \%)$ \\
Controls $(n[\%])$ & $41[29.7 \%]$ \\
Prospective $(n[\%])$ & $48(34.8 \%)$ \\
Randomized $(n[\%])$ & $1(0.7 \%)$ \\
\hline
\end{tabular}

One factor associated with higher quality research is academic collaboration. Our data suggest that while studies produced by collaboration between LMIC and non-LMIC investigators account for less than $15 \%$ of total research, they are associated with higher levels of evidence and more prospective, controlled designs.

Our finding that orthopaedic research conducted in LMICs was unlikely to attain high levels of evidence is similar to the literature published in major Western journals. For instance, Reich et al. evaluated seven years' worth of publications from The Journal of Bone and Joint Surgery (American) and found that only one of the Level 1 and 2 studies published was conducted in an LMIC [12]. In addition, the proportion of high level studies published in LMICs does not compare favorably with that of non-LMIC studies. An analysis of three American journals found that $23 \%$ of studies published in the last decade achieved Level 1 or 2 evidence [9], more than double the proportion of Level 1 and 2 LMIC-conducted studies during the same time frame. Notably, our data also suggests that the overall quality of LMIC publications has not improved over the past decade, which lies in stark contrast to studies of high impact journals that suggest the number of high-quality studies has increased significantly over time [9, 12]. Future research into why a significant gap in research quality exists between high-resource and low-resource environments is warranted.

Even though the quality of research from LMICs is lower overall, studies conducted in South Asia and Sub-Saharan Africa were more likely to attain high levels of evidence. Notably, Pakistan, India, Nigeria and Nepal produced over twothirds of Level 1 and 2 studies in the past decade. In contrast, 59 of the world's 82 LMICs did not produce a single Level 1 or 2 study over the same time frame. This finding is similar to recent literature that indirectly suggests LMICs from South Asia produce the highest quality studies in Western journals compared to other LMIC regions [12]. However, it is still unknown why this disparity by region exists, which warrants further investigation.
Collaboration between LMIC and non-LMIC investigators is associated with significantly higher quality of published clinical research with respect to levels of evidence, prospective design and presence of control groups. Notably, over $88 \%$ of these studies represented academic partnerships, in which both the LMIC and non-LMIC investigators were affiliated with an academic institution. Although, it is unclear why Collaborative studies are associated with higher research quality, recent literature has hypothesized that investigators from high-income countries can enable their counterparts in LMICs to conduct research through longitudinal partnership [4, 13, 24]. For example, Morshed et al. outline a potential model of partnership in which investigators from an academic HIC institution helped LMIC-based surgeons develop a prospective cohort study through assistance in protocol development, research training, funding and resource procurement [4]. Academic affiliation of LMIC partners may also be an important ingredient for successful partnerships, since academic advancement and research requirement for trainees are often cited by LMIC authors as incentives for research participation [4, 14].

Our study had several limitations. First, given the heterogeneity of included articles, we were unable to apply additional standardized quality assessment tools, which may have affected our ability to gauge the study quality. However, LOE has been shown to be a validated measure of study quality and have adequate interobserver reliability $[9,21]$. Second, our review only included studies since June 2004 and could have been strengthened by including more publication years. However, our study provides a snapshot of study quality over the last decade and is by far the most comprehensive existing review of the global orthopaedics literature. Third, as a literature review, our study design cannot prove causal inference exists between academic collaboration and study quality. Our data does, however, show that non-LMIC and LMIC collaboration is associated with higher study quality, which warrants further investigation. Finally, our study did not include primarily non-English publications, which may have excluded highquality studies published in other languages. However, recent literature suggests that language restrictions of reviews may not produce meaningful bias [25], although future studies with broader language requirements are needed.

\section{Conclusion}

Recent literature has shown that many investigators who practice in LMICs have a strong interest in participating in research studies [14]. However, surgeons in LMICs contend with a lack of formalized research training, resources and protected time as significant barriers to improving study quality $[4,14]$. These barriers may explain why clinical orthopaedic research conducted in LMICs rarely attains high levels of evidence compared to those conducted in non-LMICs, a phenomenon that has not improved in the past decade. Thus, more research is needed to determine how LMIC investigators can best overcome impediments to high-quality research in resource-poor environments. Academic collaboration is one of the most promising solutions, as it is associated with higher quality studies published over the last decade. Thus, greater 
investment of resources into developing and investigating the impact of academic collaboration is warranted.

\section{Funding}

No funding to disclose.

\section{Conflict of interest}

Dr. Coughlin reports AAOS-International Committee Institute for Global Orthopaedics and Traumatology/UCSF: Board or committee member.

Dr. Morshed reports personal fees from Microbion, grants from Philips outside the submitted work; Orthopaedic Research Society: Board or committee member; Orthopaedic Trauma Association: Board or committee member.

Dr. Miclau reports personal fees from Acelity, personal fees from Amgen Co, grants from Baxter, grants from Synthes outside the submitted work; Inman Abbott Society: Board or committee member; Orthopaedic Research Society: Board or committee member; Orthopaedic Trauma Association: Board or committee member; Osteosynthesis and Trauma Care Foundation: Board or committee member.

The other co-authors of this study declare no conflict of interest for this project.

Acknowledgements. The authors would like to acknowledge Dr. Edmund Eliezer and Billy Haonga from the Muhimbili Orthopaedic Institute, Dar es Salaam, Tanzania, as well as Dr. Luke Harmon, Dr. Iain Elliott, Dr. Jeremie Larouche and Dr. Brian Cunningham for their contributions to this study.

\section{References}

1. World Health Organization (2015) Global status report on road safety time for action. Available at: http:// whqlibdoc.who.int/publications/2009/9789241563840_eng.pdf. Accessed November 10, 2015.

2. Gosselin RA, Spiegel DA, Coughlin R, Zirkle LG (2009) Injuries: the neglected burden in developing countries. Bulletin of the WHO 87, 246.

3. Krug E (2012) Decade of action for road safety 2011-2020. Injury $43,6-7$.

4. Morshed S, Shearer DW, Coughlin RR. 2013 (Collaborative partnerships and the future of global orthopaedics) Clin Orthop Relat Res 471, 3088-3092.

5. Chen AT, Pedtke A, Kobs JK, Edwards GS Jr, Coughlin RR, Gosselin RA (2012) Volunteer orthopedic surgical trips in Nicaragua: a cost-effectiveness evaluation. World J Surg 36(12), 2802-2808.

6. Dormans JP, Fisher RC, Pill SG (2001) Orthopaedics in the developing world: present and future concerns. J Am Acad Orthop Surg 9(5), 289-296.
7. Museru LM, McHaro CN. 2002 (The dilemma of fracture treatment in developing countries) Int Orthop 26(6), 324-327.

8. Bernstein J (2004) Evidence-based medicine. J Am Acad Orthop Surg 12, 80-88.

9. Grant HS, Tjoumakaris FP, Maltenfort MG, Freedman KB (2014) Levels of evidence in the clinical sports medicine literature: are we getting better over time? Am J Sports Med 42(7), 1738-1742.

10. Sackett DL, Rosenberg WM, Gray JA, Haynes RB, Richardson WS (1996) Evidence based medicine: what it is and what it isn't. BMJ 312(7023), 71-72.

11. Burns PB, Rohrich RJ, Chung KC (2012) The levels of evidence and their role in evidence-based medicine. Plast Reconstr Surg 128(1), 305-310.

12. Reich MS, Shaw J, Barrett I, Goldberg VM, Schnaser E (2014) Level of evidence trends in the Journal of Bone and Joint Surgery, 1980-2010. Iowa Orthop J 34, 197-203.

13. Niles SE, Balazs GC, Cawley C, Bosse M, Mackenzie E, Li Y, Andersen RC (2015) Translating research into practice: is evidence-based medicine being practiced in military-relevant orthopedic trauma? Mil Med 180(4), 445-453.

14. Elliott IS, Sonshine DB, Akhavan S, Slade Shantz A, Caldwell A, Slade Shantz J, Gosselin RA, Coughlin RR (2015) What factors influence the production of orthopaedic research in East Africa? A qualitative analysis of interviews. Clin Orthop Relat Res 473(6), 2120-2130.

15. Arksey H, O'Malley L (2005) Scoping studies: towards a methodological framework. International Journal of Social Research Methodology: Theory \& Practice 8, 19-32.

16. Daudt HM, van Mossel C, Scott SJ (2013) Enhancing the scoping study methodology: a large, inter-professional team's experience with Arksey and O'Malley's framework. BMC Med Res Methodol 13, 48.

17. Levac D, Colquhoun H, O'Brien KK. 2010. Scoping studies: advancing the methodology. Implement Sci 5, 69.

18. World Bank (2014) http://data.worldbank.org/about/countryand-lending-groups. Accessed June 30, 2014.

19. Marx RG, Wilson SM, Swiontkowski MF (2015) Updating the assignment of levels of evidence. J Bone Joint Surg Am 97(1), $1-2$.

20. Wright JG, Swiontkowski MF, Heckman JD (2003) Introducing levels of evidence to the journal. J Bone Joint Surg Am 85, 1-3.

21. Cunningham BP, Harmsen S, Kweon C, Patterson J, Waldrop R, McLaren A, McLemore R (2013) Have levels of evidence improved the quality of orthopaedic research? Clin Orthop Relat Res 471(11), 3679-3686.

22. Murphy RF, Cibulas AM, Sawyer JR, Spence DD, Kelly DM (2015) Levels of evidence in the Journal of Pediatric Orthopaedics: update and comparison to the Journal of Bone and Joint Surgery. J Pediatr Orthop 35(7), 779-781.

23. Yarascavitch BA, Chuback JE, Almenawer SA, Reddy K, Bhandari M (2012) Levels of evidence in the neurosurgical literature: more tribulations than trials. Neurosurgery 71(6), 1131-1137, discussion 1137-8. 
24. Phillips J, Jergesen HE, Caldwell AM, Coughlin RR (2009) IGOT-The Institute for Global Orthopaedics and Traumatology: a model for collaboration and change. Tech Orthop 24(4), $308-11$.
25. Moher D, Pham B, Lawson ML, Klassen TP (2003) The inclusion of reports of randomised trials published in languages other than English in systematic reviews. Health Technol Assess 7(41), 1-90.

Cite this article as: Wu H-H, Liu M, Patel KR, Turner W, Baltus L, Caldwell AM, Hahn JC, Coughlin RR, Morshed S, Miclau T \& Shearer DW (2017) Impact of academic collaboration and quality of clinical orthopaedic research conducted in low- and middle-income countries. SICOT J, 3, 6

\section{Appendix}

\section{Search strategy}

1. orthopedics[mh] OR orthopedic procedures[mh] OR fractures, bone[mh] OR musculoskeletal diseases[mh:noexp] OR bone diseases[mh] OR cartilage diseases[mh:noexp] OR chondromalacia patellae[mh] OR osteochondritis[mh] OR polychondritis, relapsing[mh] OR foot deformities[mh] OR foot diseases[mh] OR hand deformities[mh] OR joint diseases[mh] OR muscular diseases[mh:noexp] OR arthrogryposis[mh] OR compartment syndromes[mh] OR contracture[mh] OR medial tibial stress syndrome[mh] OR musculoskeletal pain[mh] OR tendinopathy[mh] OR musculoskeletal abnormalities[mh:noexp] OR campomelic dysplasia[mh] OR hip dislocation, congenital[mh] OR klippel-feil syndrome[mh] OR limb deformities, congenital[mh] OR synostosis[mh] OR rheumatic diseases[mh:noexp] OR osteoarthritis[mh] OR tennis elbow[mh] OR amputation, traumatic[mh] OR arm injuries[mh] OR back injuries[mh] OR craniocerebral trauma[mh:noexp] OR dislocations[mh] OR fractures, cartilage[mh] OR hand injuries[mh] OR hip injuries[mh] OR leg injuries[mh] OR ligaments, articular[majr] OR limb salvage[mh] OR muscle, skeletal[majr] OR musculoskeletal system[mh:noexp] OR orthopedic equipment[mh] OR skeleton/injuries[mh] OR skeleton/surgery[mh] OR spinal injuries[mh] OR spine/injuries[mh] OR tendon injuries[mh] OR tendons[majr]

2. amput*[tiab] OR dislocation*[tiab] OR fracture[tiab] OR fractures[tiab] OR musculoskeletal*[tiab] OR orthoped*[tiab] OR orthopaed*[tiab]

3. accidents[mh] OR accident*[tiab] OR injur*[tiab] OR polytrauma*[tiab] OR "wounds and injuries"[majr:noexp] OR wounds, gunshot[mh] OR blast injuries[mh]

4. bone[tiab] OR bones[tiab] OR humerus[tiab] OR humeri[tiab] OR ulna[tiab] OR ulnas[tiab] OR ulnae [tiab] OR scaphoid[tiab] OR scaphoids[tiab] OR vertebra*[tiab] OR spine[tiab] OR spines[tiab] OR pelvis* [tiab] OR pelves[tiab] OR femur[tiab] OR femurs[tiab] OR tibia[tiab] OR tibias[tiab] OR fibula[tiab] OR fibulas[tiab] OR talus[tiab] OR tali[tiab] OR calcaneus[tiab] OR calcanei[tiab] OR calcanea[tiab] OR shoulder[tiab] OR shoulders[tiab] OR elbow[tiab] OR elbows[tiab] OR wrist[tiab] OR wrists[tiab] OR hip[tiab] OR hips [tiab] OR knee[tiab] OR knees[tiab] OR ankle[tiab] OR ankles[tiab] OR extremity[tiab] OR extremities[tiab] OR "open injury" [tiab] OR "open injuries"[tiab]

5. 3 AND 4

6. 1 OR 2 OR 5

7. Afghanistan[Mesh] OR Bangladesh[Mesh] OR Benin [Mesh] OR Burkina Faso[Mesh] OR Burundi[Mesh] OR Cambodia[Mesh] OR Central African Republic[Mesh] OR Chad[Mesh] OR Comoros[Mesh] OR Democratic Republic of the Congo[Mesh] OR Eritrea [Mesh] OR Ethiopia[Mesh] OR Gambia[Mesh] OR Guinea[Mesh] OR Guinea-Bissau[Mesh] OR Haiti[Mesh] OR Kenya[Mesh] OR Democratic People's Republic of Korea[Mesh] OR Kyrgyzstan[Mesh] OR Liberia[Mesh] OR Madagascar[Mesh] OR Malawi [Mesh] OR Mali[mh] OR Mozambique[Mesh] OR Myanmar[Mesh] OR Nepal[Mesh] OR Niger[Mesh] OR Rwanda[Mesh] OR Sierra Leone[Mesh] OR Somalia[Mesh] OR Sudan[Mesh] OR Tajikistan[Mesh] OR Tanzania[Mesh] OR Togo[Mesh] OR Uganda[Mesh] OR Zimbabwe[Mesh]

8. Armenia[Mesh] OR Bhutan[Mesh] OR Bolivia[Mesh] OR Cameroon[Mesh] OR Cape Verde[Mesh] OR Congo[Mesh] OR Cote d'Ivoire[Mesh] OR Djibouti[Mesh] OR Egypt[Mesh] OR El Salvador[Mesh] OR "Georgia (Republic)"[Mesh] OR Ghana[Mesh] OR Guatemala [Mesh] OR Guyana[Mesh] OR Honduras[Mesh] OR Indonesia[Mesh] OR India[Mesh] OR Kosovo[Mesh] OR Laos[Mesh] OR Lesotho[Mesh] OR Mauritania[Mesh] OR Moldova[Mesh] OR Mongolia[Mesh] OR Morocco[Mesh] OR Nicaragua[Mesh] OR Nigeria[Mesh] OR Pakistan[Mesh] OR Papua New Guinea[Mesh] OR Paraguay[Mesh] OR Philippines[Mesh] OR Samoa[Mesh:noexp] OR Independent State of Samoa[mh] OR Senegal[Mesh] OR Sri Lanka[Mesh] OR Sudan[Mesh] OR Swaziland[Mesh] OR Syria[Mesh] OR East Timor[Mesh] OR Ukraine[Mesh] OR Uzbekistan[Mesh] OR Vanuatu[Mesh] OR (Vietnam[Mesh] NOT veteran*) OR Yemen[Mesh] OR Zambia[Mesh]

9. Afghani*[tiab] OR Bangladesh*[tiab] OR Benin[tiab] OR "Burkina Faso" [tiab] OR Burundi*[tiab] OR Cambodia*[tiab] OR "Central African Republic"[tiab] OR Chad[tiab] OR Comoros[tiab] OR (Congo[tiab] NOT "congo red") OR Congolese[tiab] OR Zaire[tiab] OR Eritrea*[tiab] OR Ethiopia*[tiab] OR Gambia*[tiab] OR (Guinea*[tiab] NOT (guinea fowl* OR guinea pig* OR "new guinea")) OR "Guinea-Bissau"[tiab] OR 
Haiti*[tiab] OR Kenya*[tiab] OR “Democratic People's Republic of Korea"[tiab] OR "North Korea"[tiab] OR North Korean*[tiab] OR Kyrgyz*[tiab] OR Liberia* [tiab] OR Madagascar[tiab] OR Malawi[tiab] OR Malawian*[tiab] OR Mali[tiab] OR Mozambique[tiab] OR Myanmar[tiab] OR Burma[tiab] OR Burmese[tiab] OR Nepal[tiab] OR Nepalese[tiab] OR Niger[tiab] OR Rwanda*[tiab] OR "Sierra Leone"[tiab] OR Somalia [tiab] OR Somali[tiab] OR Somalis[tiab] OR "South Sudan"[tiab] OR Tajikistan*[tiab] OR Tadjikistan*[tiab] OR Tanzania*[tiab] OR Zanzibar*[tiab] OR Tanganyika[tiab] OR Togo[tiab] OR Togolese[tiab] OR Uganda*[tiab] OR Zimbabw*[tiab] OR (Rhodesia[tiab] NOT "Rhodesian ridgeback")

10. Armenia*[tiab] OR Bhutan*[tiab] OR Bolivia*[tiab] OR Cameroon*[tiab] OR "Cape Verde"[tiab] OR "Cote d'Ivoire"[tiab] OR "Ivory Coast"[tiab] OR Djibouti* [tiab] OR Egypt*[tiab] OR El Salvador*[tiab] OR "Georgia Republic"[tiab] OR "Republic of Georgia" [tiab] OR Ghana*[tiab] OR Guatemal*[tiab] OR Guyana[tiab] OR Guyanese[tiab] OR "British Guiana" [tiab] OR Hondur*[tiab] OR (India[tiab] NOT ("india ink" OR “indian ink")) OR “Asian Indian”[tiab] OR "Asian Indians"[tiab] OR Indonesia*[tiab] OR Kiribati[tiab] OR Kosovo[tiab] OR Kosovan*[tiab] OR Kosovar*[tiab] OR Laos[tiab] OR "Lao PDR" OR “LAO People's Democratic Republic"[tiab] OR Laotian*[tiab] OR Lesotho*[tiab] OR Mauritania*[tiab] OR (Micronesia*[tiab] AND "Federated States"[tiab]) OR Moldova*[tiab] OR Mongolia*[tiab] OR Morocc*[tiab] OR Nicaragua*[tiab] OR Nigeria*[tiab] OR Pakistan* [tiab] OR "Papua New Guinea"[tiab] OR Paraguay[tiab] OR Paraguayan*[tiab] OR Philippines[tiab] OR (Filipino*[tiab] OR Filipina*[tiab] NOT (Filipino American* OR United States[mh])) OR (Samoa*[tiab] NOT American Samoa*) OR "Sao Tome"[tiab] OR Senegal[tiab] OR Senegalese[tiab] OR Solomon Island*[tiab] OR "Sri Lanka"[tiab] OR Sri Lankan* [tiab] OR Ceylon[tiab] OR Sudan*[tiab] OR Swaziland [tiab] OR Syria*[tiab] OR “Timor-Leste"[tiab] OR "East Timor"[tiab] OR Ukrain*[tiab] OR Uzbekistan* [tiab] OR Vanuatu*[tiab] OR (Vietnam*[tiab] NOT veteran*) OR "West Bank"[tiab] OR Gaza[tiab] OR Palestin*[tiab] OR Yemen*[tiab] OR Zambia*[tiab]

11. developing countries[mh] OR "developing country" [tiab] OR "developing countries"[tiab] OR LMIC[tiab] OR LMICs[tiab] OR "low income countries"[tiab] OR "low income country"[tiab] OR "low and middle income countries"[tiab] OR "low and middle income country"[tiab] OR "lower middle income countries" [tiab] OR "lower middle income country" [tiab] OR “developing nation"[tiab] OR "developing nations"[tiab] OR "developing world"[tiab] OR "developing economy"[tiab] OR "developing economies"[tiab] OR "transitional country"[tiab] OR "transitional countries"[tiab] OR "global burden"[tiab] OR "global health"[tiab] OR global orthop*[tiab] OR "global outreach"[tiab] OR "global public health"[tiab] OR (global[ti] AND watch[ti]) OR “international health"[tiab] OR "international public health"[tiab] OR world health[majr] OR international cooperation [majr] OR "resource poor" [tiab] OR austere environment*[tiab] OR "third world" [tiab]

12. Africa[mh:noexp] OR Africa, Central[mh:noexp] OR Africa, Eastern[mh:noexp] OR Africa, Northern[mh:noexp] OR Africa South of the Sahara[mh:noexp] OR Africa, Southern[mh:noexp] OR Africa, Western[mh:noexp] OR central Africa*[ti] OR east Africa*[ti] OR eastern Africa*[ti] OR north Africa*[ti] OR northern Africa*[ti] OR southern Africa*[ti] OR west africa*[ti] OR western africa*[ti] OR sahara*[ti] OR subsahara*[ti] OR (Asia[mh:noexp] NOT (china[mh] OR japan[mh] OR Singapore[mh] OR south korea[mh])) OR Asia, Central[mh:noexp] OR Asia, Southeastern[mh:noexp] OR Asia, Western[mh:noexp] OR central asia*[ti] OR south asia*[ti] OR south asia*[ti] OR southeast asia*[ti] OR southeastern asia*[ti] OR southern asia*[ti] OR west asia*[tiab] OR western asia*[ti] OR Central America[mh:noexp] OR central America*[ti] OR Europe, Eastern[mh:noexp] OR eastern Europe*[ti] OR South America[mh:noexp] OR South America*[ti] OR Caribbean Region[mh:noexp] OR Caribbean[ti] OR Middle East[mh:noexp]

13. 7 OR 8 OR 9 OR 10 OR 11 OR 12

14. 7 OR 8 OR 9 OR 10 OR 11 OR 12 NOT (animals[mh] NOT humans[mh]) NOT Dental journals[sb] NOT News[pt] NOT (mummies OR mummy OR history, ancient[mh] OR paleoanthro* OR paleoepidem* OR paleopath* OR paleont* OR archeolog* OR ancient egypt* OR dynast* OR fossil* OR forensic anthropology[mh] OR history of medicine[mh])

15. 14 AND ("2004”[Date - Publication]: “3000”[Date Publication])

16. 14 AND ("2004"[Date - Publication]: “3000"[Date Publication]) AND English[Language]

\section{Pubmed and Cochrane Search (June 1, 2014)}

(orthopedics[mh] OR orthopedic procedures[mh] OR fractures, bone[mh] OR musculoskeletal diseases[mh:noexp] OR bone diseases[mh] OR cartilage diseases[mh:noexp] OR chondromalacia patellae[mh] OR osteochondritis[mh] OR polychondritis, relapsing[mh] OR foot deformities[mh] OR foot diseases[mh] OR hand deformities[mh] OR joint diseases[mh] OR muscular diseases[mh:noexp] OR arthrogryposis[mh] OR compartment syndromes[mh] OR contracture[mh] OR medial tibial stress syndrome[mh] OR musculoskeletal pain[mh] OR tendinopathy[mh] OR musculoskeletal abnormalities[mh:noexp] OR campomelic dysplasia[mh] OR hip dislocation, congenital[mh] OR klippel-feil syndrome[mh] OR limb deformities, congenital[mh] OR synostosis[mh] OR rheumatic diseases[mh:noexp] OR osteoarthritis[mh] OR tennis elbow[mh] OR amputation, traumatic[mh] OR arm injuries[mh] OR back injuries[mh] OR craniocerebral trauma [mh:noexp] OR dislocations[mh] OR fractures, cartilage[mh] OR hand injuries[mh] OR hip injuries[mh] OR leg injuries[mh] OR ligaments, articular[majr] OR limb salvage[mh] 
OR muscle, skeletal[majr] OR musculoskeletal system[mh:noexp] OR orthopedic equipment[mh] OR skeleton/injuries[mh] OR skeleton/surgery[mh] OR spinal injuries[mh] OR spine/injuries[mh] OR tendon injuries[mh] OR tendons[majr] OR amput*[tiab] OR dislocation*[tiab] OR fracture[tiab] OR fractures[tiab] OR musculoskeletal*[tiab] OR orthoped*[tiab] OR orthopaed*[tiab] OR ((accidents[mh] OR accident*[tiab] OR injur*[tiab] OR polytrauma*[tiab] OR "wounds and injuries" [majr:noexp] OR wounds, gunshot[mh] OR blast injuries[mh]) AND (bone[tiab] OR bones[tiab] OR humerus[tiab] OR humeri[tiab] OR ulna[tiab] OR ulnas[tiab] OR ulnae[tiab] OR scaphoid[tiab] OR scaphoids[tiab] OR vertebra*[tiab] OR spine[tiab] OR spines[tiab] OR pelvis*[tiab] OR pelves [tiab] OR femur[tiab] OR femurs[tiab] OR tibia[tiab] OR tibias[tiab] OR fibula[tiab] OR fibulas[tiab] OR talus[tiab] OR tali[tiab] OR calcaneus[tiab] OR calcanei[tiab] OR calcanea[tiab] OR shoulder[tiab] OR shoulders[tiab] OR elbow[tiab] OR elbows[tiab] OR wrist[tiab] OR wrists[tiab] OR hip[tiab] OR hips[tiab] OR knee[tiab] OR knees[tiab] OR ankle[tiab] OR ankles[tiab] OR extremity[tiab] OR extremities[tiab] OR "open injury"[tiab] OR “open injuries"[tiab]))) AND ((Afghanistan[Mesh] OR Bangladesh[Mesh] OR Benin[Mesh] OR Burkina Faso[Mesh] OR Burundi[Mesh] OR Cambodia[Mesh] OR Central African Republic[Mesh] OR Chad[Mesh] OR Comoros[Mesh] OR Democratic Republic of the Congo[Mesh] OR Eritrea[Mesh] OR Ethiopia[Mesh] OR Gambia[Mesh] OR Guinea[Mesh] OR Guinea-Bissau[Mesh] OR Haiti[Mesh] OR Kenya[Mesh] OR Democratic People's Republic of Korea [Mesh] OR Kyrgyzstan[Mesh] OR Liberia[Mesh] OR Madagascar[Mesh] OR Malawi[Mesh] OR Mali[mh] OR Mozambique[Mesh] OR Myanmar[Mesh] OR Nepal[Mesh] OR Niger[Mesh] OR Rwanda[Mesh] OR Sierra Leone[Mesh] OR Somalia[Mesh] OR Sudan[Mesh] OR Tajikistan[Mesh] OR Tanzania[Mesh] OR Togo[Mesh] OR Uganda[Mesh] OR Zimbabwe[Mesh] OR Armenia[Mesh] OR Bhutan[Mesh] OR Bolivia[Mesh] OR Cameroon[Mesh] OR Cape Verde[Mesh] OR Congo[Mesh] OR Cote d'Ivoire[Mesh] OR Djibouti [Mesh] OR Egypt[Mesh] OR El Salvador[Mesh] OR "Georgia (Republic)"[Mesh] OR Ghana[Mesh] OR Guatemala[Mesh] OR Guyana[Mesh] OR Honduras[Mesh] OR Indonesia[Mesh] OR India[Mesh] OR Kosovo[Mesh] OR Laos[Mesh] OR Lesotho[Mesh] OR Mauritania[Mesh] OR Moldova[Mesh] OR Mongolia[Mesh] OR Morocco[Mesh] OR Nicaragua [Mesh] OR Nigeria[Mesh] OR Pakistan[Mesh] OR Papua New Guinea[Mesh] OR Paraguay[Mesh] OR Philippines[Mesh] OR Samoa[Mesh:noexp] OR Independent State of Samoa[mh] OR Senegal[Mesh] OR Sri Lanka[Mesh] OR Sudan[Mesh] OR Swaziland[Mesh] OR Syria[Mesh] OR East Timor[Mesh] OR Ukraine[Mesh] OR Uzbekistan[Mesh] OR Vanuatu[Mesh] OR (Vietnam[Mesh] NOT veteran*) OR Yemen[Mesh] OR Zambia[Mesh] OR Afghani*[tiab] OR Bangladesh*[tiab] OR Benin[tiab] OR "Burkina Faso"[tiab] OR Burundi*[tiab] OR Cambodia*[tiab] OR "Central African Republic"[tiab] OR Chad[tiab] OR Comoros[tiab] OR (Congo[tiab] NOT "congo red") OR Congolese[tiab] OR Zaire[tiab] OR Eritrea*[tiab] OR Ethiopia*[tiab] OR Gambia*[tiab] OR (Guinea*[tiab] NOT (guinea fowl* OR guinea pig* OR "new guinea")) OR “Guinea-Bissau"[tiab] OR Haiti*[tiab] OR Kenya*[tiab] OR "Democratic People's
Republic of Korea"[tiab] OR "North Korea"[tiab] OR North Korean*[tiab] OR Kyrgyz*[tiab] OR Liberia*[tiab] OR Madagascar[tiab] OR Malawi[tiab] OR Malawian*[tiab] OR Mali [tiab] OR Mozambique[tiab] OR Myanmar[tiab] OR Burma[tiab] OR Burmese[tiab] OR Nepal[tiab] OR Nepalese[tiab] OR Niger[tiab] OR Rwanda*[tiab] OR "Sierra Leone"[tiab] OR Somalia[tiab] OR Somali[tiab] OR Somalis[tiab] OR "South Sudan"[tiab] OR Tajikistan*[tiab] OR Tadjikistan*[tiab] OR Tanzania*[tiab] OR Zanzibar*[tiab] OR Tanganyika[tiab] OR Togo[tiab] OR Togolese[tiab] OR Uganda*[tiab] OR Zimbabw*[tiab] OR (Rhodesia[tiab] NOT “Rhodesian ridgeback") OR Armenia*[tiab] OR Bhutan*[tiab] OR Bolivia*[tiab] OR Cameroon*[tiab] OR "Cape Verde"[tiab] OR "Cote d'Ivoire"[tiab] OR "Ivory Coast"[tiab] OR Djibouti*[tiab] OR Egypt*[tiab] OR El Salvador*[tiab] OR “Georgia Republic"[tiab] OR "Republic of Georgia"[tiab] OR Ghana*[tiab] OR Guatemal*[tiab] OR Guyana[tiab] OR Guyanese[tiab] OR "British Guiana"[tiab] OR Hondur*[tiab] OR (India[tiab] NOT ("india ink" OR "indian ink")) OR "Asian Indian"[tiab] OR “Asian Indians"[tiab] OR Indonesia*[tiab] OR Kiribati [tiab] OR Kosovo[tiab] OR Kosovan*[tiab] OR Kosovar*[tiab] OR Laos[tiab] OR “Lao PDR" OR "LAO People's Democratic Republic"[tiab] OR Laotian*[tiab] OR Lesotho*[tiab] OR Mauritania*[tiab] OR (Micronesia*[tiab] AND "Federated States"[tiab]) OR Moldova*[tiab] OR Mongolia*[tiab] OR Morocc*[tiab] OR Nicaragua*[tiab] OR Nigeria*[tiab] OR Pakistan*[tiab] OR "Papua New Guinea" [tiab] OR Paraguay [tiab] OR Paraguayan*[tiab] OR Philippines[tiab] OR (Filipino*[tiab] OR Filipina*[tiab] NOT (Filipino American* OR United States[mh])) OR (Samo**tiab] NOT American Samoa*) OR "Sao Tome"[tiab] OR Senegal[tiab] OR Senegalese[tiab] OR Solomon Island*[tiab] OR "Sri Lanka"[tiab] OR Sri Lankan*[tiab] OR Ceylon[tiab] OR Sudan*[tiab] OR Swaziland[tiab] OR Syria*[tiab] OR “Timor-Leste"[tiab] OR "East Timor"[tiab] OR Ukrain*[tiab] OR Uzbekistan*[tiab] OR Vanuatu*[tiab] OR (Vietnam*[tiab] NOT veteran*) OR "West Bank"[tiab] OR Gaza[tiab] OR Palestin*[tiab] OR Yemen*[tiab] OR Zambia*[tiab]) OR (developing countries[mh] OR “developing country"[tiab] OR “developing countries"[tiab] OR LMIC[tiab] OR LMICs[tiab] OR "low income countries"[tiab] OR "low income country"[tiab] OR "low and middle income countries"[tiab] OR "low and middle income country" [tiab] OR "lower middle income countries" [tiab] OR "lower middle income country"[tiab] OR "developing nation" [tiab] OR “developing nations" [tiab] OR “developing world"[tiab] OR "developing economy"[tiab] OR "developing economies"[tiab] OR "transitional country"[tiab] OR "transitional countries"[tiab] OR "global burden"[tiab] OR "global health" [tiab] OR global orthop*[tiab] OR "global outreach"[tiab] OR "global public health"[tiab] OR (global[ti] AND watch[ti]) OR “international health" [tiab] OR "international public health" [tiab] OR world health[majr] OR international cooperation[majr] OR "resource poor"[tiab] OR austere environment*[tiab] OR "third world"[tiab]) OR (Africa[mh:noexp] OR Africa, Central[mh:noexp] OR Africa, Eastern[mh:noexp] OR Africa, Northern[mh:noexp] OR Africa South of the Sahara[mh:noexp] OR Africa, Southern[mh: noexp] OR Africa, Western[mh:noexp] OR central Africa*[ti] OR east Africa*[ti] OR eastern Africa*[ti] OR north 
Africa*[ti] OR northern Africa*[ti] OR southern Africa*[ti] OR west africa*[ti] OR western africa*[ti] OR sahara*[ti] OR subsahara*[ti] OR (Asia[mh:noexp] NOT (china[mh] OR japan [mh] OR Singapore[mh] OR south korea[mh])) OR Asia, Central[mh:noexp] OR Asia, Southeastern[mh:noexp] OR Asia, Western[mh:noexp] OR central asia*[i] OR south asia*[ti] OR south asia*[ti] OR southeast asia*[ti] OR southeastern asia*[it] OR southern asia*[ti] OR west asia*[tiab] OR western asia*[ti] OR Central America[mh:noexp] OR central America*[ti] OR Europe, Eastern[mh:noexp] OR eastern Europe*[ti] OR South America[mh:noexp] OR South America*[ti] OR Caribbean Region[mh:noexp] OR Caribbean[ti] OR Middle East[mh:noexp])) NOT (animals[mh] NOT humans[mh]) NOT Dental journals[sb] NOT News[pt] NOT (mummies OR mummy OR history, ancient[mh] OR paleoanthro* OR paleoepidem* OR paleopath* OR paleont* OR archeolog* OR ancient egypt* OR dynast* OR fossil* OR forensic anthropology[mh] OR history of medicine[mh]) AND ("2004”[Date - Publication]: "3000”[Date - Publication]) AND English[Language].

\section{Embase Search (June 1, 2014)}

'orthopedics'/de OR 'orthopedic surgery'/exp OR 'fracture'/exp OR 'musculoskeletal disease'/de OR 'arthropathy'/exp OR 'bone disease'/exp OR 'chondropathy'/exp OR 'compartment syndrome'/exp OR 'contracture'/mj OR 'flexion contracture'/de OR 'hip contracture'/de OR 'joint contracture'/ de OR 'muscle contracture'/de OR 'tendon contracture'/de OR 'dupuytren contracture'/de OR 'enthesopathy'/exp OR 'limb disease'/de OR 'arm disease'/exp OR 'leg disease'/exp OR 'limb defect'/de OR 'limb deformity'/de OR 'limb injury'/exp OR 'limb malformation'/exp OR 'limb pain'/de OR 'limb tumor'/de OR 'muscle disease'/mj OR 'musculoskeletal injury'/exp OR 'musculoskeletal pain'/exp OR 'musculoskeletal system malformation'/exp/mj OR 'rheumatic disease'/de OR 'tendinitis'/exp OR 'ligament'/exp/mj OR 'musculoskeletal system'/mj OR 'orthopedic equipment'/exp/mj OR 'pelvis injury'/exp OR 'skeletal muscle'/exp/mj OR 'tendon'/exp/mj OR amput*:ab,ti OR back NEAR/3 injur* OR dislocation*:ab,ti OR fracture:ab,ti OR fractures:ab,ti OR musculoskelet*:ab,ti OR orthopedi*:ab,ti OR orthopaedi*:ab,ti OR ('accident'/exp OR 'accidental injury'/de OR accident*:ab,ti OR injur*:ab,ti OR polytrauma*:ab,ti OR 'injury'/mj OR 'blunt trauma'/exp OR 'crush trauma'/de OR 'multiple trauma'/de AND (bone:ab,ti OR bones:ab,ti OR humerus:ab,ti OR humeri:ab,ti OR ulna:ab,ti OR ulnas:ab,ti OR ulnae:ab,ti OR scaphoid:ab,ti OR scaphoids:ab,ti OR vertebra*:ab,ti OR spine:ab,ti OR spines:ab,ti OR pelvis*:ab,ti OR pelves:ab,ti OR femur:ab,ti OR femurs:ab,ti OR tibia:ab,ti OR tibias:ab,ti OR fibula:ab,ti OR fibulas:ab,ti OR talus:ab,ti OR tali:ab,ti OR calcaneus:ab,ti OR calcanei:ab,ti OR calcanea:ab,ti OR shoulder:ab,ti OR shoulders:ab,ti OR elbow:it,ab OR elbows:ab,ti OR wrist:ab,ti OR wrists:ab,ti OR hip:ab,ti OR hips:ab,ti OR knee:ab,ti OR knees:ab,ti OR ankle:ab,ti OR ankles:ab,ti OR extremity:ab,ti OR extremities:ab,ti OR 'open injury':ab,ti
OR 'open injuries':ab,ti)) AND ('afghanistan'/de OR 'bangladesh'/de OR 'benin'/de OR 'burkina faso'/de OR 'burundi'/de OR 'cambodia'/de OR 'central african republic'/de OR 'chad'/ de OR 'comoros'/de OR 'democratic republic congo'/de OR 'eritrea'/de OR 'ethiopia'/de OR 'gambia'/de OR 'guinea'/de OR 'guinea-bissau'/de OR 'haiti'/de OR 'kenya'/de OR 'north korea'/de OR 'kyrgyzstan'/de OR 'liberia'/de OR 'madagascar'/de OR 'malawi'/de OR 'mali'/de OR 'mozambique'/de OR 'myanmar'/de OR 'nepal'/de OR 'niger'/de OR 'rwanda'/de OR 'sierra leone'/de OR 'somalia'/de OR 'tajikistan'/de OR 'tanzania'/de OR 'togo'/de OR 'uganda'/de OR 'zimbabwe'/de OR 'armenia'/de OR 'bhutan'/de OR 'bolivia'/de OR 'cameroon'/de OR 'cape verde'/de OR 'congo'/ de OR 'cote d'ivoire'/exp OR 'djibouti'/de OR 'egypt'/de OR 'el salvador'/de OR 'georgia (republic)'/de OR 'ghana'/ de OR 'guatemala'/de OR 'guyana'/de OR 'honduras'/de OR 'indonesia'/de OR 'india'/de OR 'kosovo'/de OR 'laos'/de OR 'lesotho'/de OR 'mauritania'/de OR 'federated states of micronesia'/de OR 'moldova'/de OR 'mongolia'/de OR 'morocco'/de OR 'nicaragua'/de OR 'nigeria'/de OR 'pakistan'/de OR 'papua new guinea'/de OR 'paraguay'/de OR 'philippines'/de OR 'samoa'/de OR 'sao tome and principe'/de OR 'senegal'/de OR 'solomon islands'/de OR 'sri lanka'/de OR 'sudan'/de OR 'swaziland'/de OR 'syrian arab republic'/de OR 'timor-leste'/de OR 'ukraine'/de OR 'uzbekistan'/de OR 'vanuatu'/de OR ('vietnam'/de NOT veteran*) OR 'palestine'/de OR 'yemen'/de OR 'zambia'/de OR afghani*:ab,ti OR bangladesh*:ab,ti OR benin:ab,ti OR 'burkina faso':ab,ti OR burundi*:ab,ti OR cambodia*:ab,ti OR 'central african republic':ab,ti OR chad:ab,ti OR comoros:ab,ti OR (congo:ab,ti NOT 'congo red') OR congolese:ab,ti OR zaire:ab,ti OR eritrea*:ab,ti OR ethiopia*:ab,ti OR gambia*:ab,ti OR (guinea*:ab,ti NOT (guinea NEXT/1 fowl* OR guinea NEXT/1 pig* OR 'guinea pig'/exp OR 'new guinea')) OR 'guinea-bissau':ab,ti OR haiti*:ab,ti OR kenya*:ab,ti OR 'north korea':ab,ti OR 'north korean':ab,ti OR 'north koreans':ab,ti OR 'kyrgyz republic':ab,ti OR kyrgyzstan*:ab,ti OR liberia*:ab,ti OR madagascar:ab,ti OR malawi:ab,ti OR maliab,ti OR mozambique:ab,ti OR myanmar:ab,ti OR burma:ab,ti OR burmese:ab,ti OR nepal:ab,ti OR nepalese:ab,ti OR niger:ab,ti OR rwanda*:ab,ti OR 'sierra leone':ab,ti OR somalia:ab,ti OR somali:ab,ti OR somalis:ab,ti OR 'south sudan':ab,ti OR tajikistan*:ab,ti OR tadjikistan*:ab,ti OR tanzania*:ab,ti OR zanzibar*:ab,ti OR tanganyika:ab,ti OR togo:ab,ti OR togolese:ab,ti OR uganda*:ab,ti OR zimbabw*:ab,ti OR (rhodesia:ab,ti NOT 'rhodesian ridgeback') OR armenia*:ab,ti OR bhutan*:ab,ti OR bolivia*ab,ti OR cameroon*:ab,ti OR 'cape verde':ab,ti OR 'cote d ivoire':ab,ti OR 'ivory coast':ab,ti OR djibouti*:ab,ti OR egypt*:ab,ti OR 'el salvador':ab,ti OR 'el salvadoran':ab,ti OR 'el salvadorans':ab,ti OR 'georgia republic':ab,ti OR 'republic of georgia':ab,ti OR ghana*:ab,ti OR guatemal*:ab,ti OR guyana:ab,ti OR guyanese:ab,ti OR 'british guiana':ab,ti OR hondur*:ab,ti OR (india:ab,ti NOT ('india ink' OR 'indian ink')) OR 'asian indian':ab,ti OR 'asian indians':ab,ti OR indonesia*:ab,ti OR kiribati:ab,ti OR kosovo:ab,ti OR 
laos:ab,ti OR 'lao pdr' OR laotian*:ab,ti OR lesotho*:ab,ti OR mauritania*:ab,ti OR (micronesia:ab,ti AND 'federated states') OR moldova:ab,ti OR mongolia*:ab,ti OR moroce*:ab,ti OR nicaragua*:ab,ti OR nigeria*:ab,ti OR pakistan*:ab,ti OR 'papua new guinea':ab,ti OR paraguay:ab,ti OR philippines:ab,ti OR (filipino*:ab,ti OR filipina*:ab,ti NOT ('filipino american' OR 'filipino americans' OR 'filipina american' OR 'filipina americans' OR 'united states'/exp)) OR (samoa:ab,ti OR samoan*:ab,ti NOT american NEXT/1 samoa*) OR 'sao tome':ab,ti OR senegal:ab,ti OR senegalese:ab,ti OR 'solomon island':ab,ti OR 'solomon islands':ab,ti OR 'sri lanka':ab,ti OR 'sri lankan':ab,ti OR 'sri lankans':ab,ti OR ceylon:ab,ti OR sudan*:ab,ti OR swaziland:ab,ti OR syria*:ab,ti OR 'timor-leste':ab,ti OR 'east timor':ab,ti OR ukrain*:ab,ti OR uzbekistan*:ab,ti OR vanuatu*:ab,ti OR (vietnam*:ab,ti NOT veteran*) OR 'west bank':ab,ti OR gaza:ab,ti OR palesti$\mathrm{n}^{*}: \mathrm{ab}$,ti OR yemen*:ab,ti OR zambia*:ab,ti OR 'developing country'/de OR 'developing country':ab,ti OR 'developing countries':ab,ti OR lmic:ab,ti OR lmics:ab,ti OR 'low income countries':ab,ti OR 'low income country':ab,ti OR 'low and middle income countries':ab,ti OR 'low and middle income country':ab,ti OR 'lower middle income countries':ab,ti OR 'lower middle income country':ab,ti OR 'developing nation':ab,ti OR 'developing nations':ab,ti OR 'developing world':ab,ti OR 'developing economy':ab,ti OR 'developing economies':ab,ti OR 'transitional country':ab,ti OR 'transitional countries':ab,ti OR 'global burden':ab,ti OR 'global health':ab,ti OR (global NEXT/1 orthop*):ab,ti OR 'global outreach':ab,ti OR 'global public health':ab,ti OR 'global watch':ab,ti OR 'international health':ab,ti OR 'international public health':ab,ti OR 'international cooperation'/mj OR 'resource poor':ab,ti OR 'austere environment':ab,ti OR 'third world':ab,ti OR 'africa'/de OR 'central africa'/de OR 'north africa'/de OR 'africa south of the sahara'/de OR (central NEXT/1 africa*):ti OR (east NEXT/1 africa*):ti OR (eastern NEXT/1 afri$\mathrm{ca}^{*}$ ):ti OR (north NEXT/1 africa*):ti OR (northern NEXT/1 africa*):ti OR (southern NEXT/1 africa*):ti OR sahara*:ti OR subsahara*:ti OR (west NEXT/1 africa*):ti OR (western NEXT/1 africa*):ti OR ('asia'/mj NOT ('china'/de OR 'japan'/de OR 'singapore'/de OR 'south korea'/de)) OR 'south asia'/de OR 'southeastern asia' OR (central NEXT/1 asia*):ti OR (south NEXT/1 asia*):ti OR (southeast* NEXT/1 asia*):ti OR (southern NEXT/1 asia*):ti OR (west NEXT/1 asia*):ti OR (western NEXT/1 asia*):ti OR 'central america'/de OR (central NEXT/1 america*):ti OR 'eastern europe'/de OR (eastern NEXT/1 europe*):ti OR 'south america'/de OR 'south and central america'/de OR (south NEXT/1 america*):ti OR 'caribbean islands'/de OR caribbean:ti OR 'middle east'/de) NOT ([animals]/lim NOT ([humans]/lim OR 'patient'/exp)) NOT ('mummies'/exp OR 'mummy'/exp OR 'ancient history' OR paleo* OR archeolog* OR ancient NEXT/1 egypt* OR dynast* OR 'forensic anthropology'/exp OR 'history of medicine'/exp OR 'history'/exp) AND [english]/lim AND [2004-2014]/py.

\section{Cochrane Library Search (June 1, 2014)}

orthop* or fracture* or musculoskeletal or amput* or dislocat* or extremity or extremities or bone or bones or ankle or calcaneus or elbow or femur or fibula or femur or foot or hand or hip or humerus or knee or leg or pelvis or scaphoid or shoulder or spine or talus or tibia or ulna or vertebrae or wrist or open next injur*:ti,ab,kw and Afghani* or Bangladesh* or Benin or "Burkina Faso" or Burundi* or Cambodia* or "Central African Republic" or Chad or Comoros or (Congo* not "congo red") or Zaire or Eritrea* or Ethiopia* or Gambia* or (Guinea not (guinea next fowl* or guinea next pig* or "new guinea")) or "Guinea-Bissau" or Haiti* or Kenya* or (Democratic next People* and "Republic of Korea") or North next Korea* or Kyrgyz* or Liberia or Madagascar or Malawi* or Mali or Mozambique or Myanmar or Burma or Burmese or Nepal or Nepalese or Niger or Rwanda* or "Sierra Leone" or Somali* or "South Sudan" or Tajikistan* or Tadjikistan* or Tanzania* or Zanzibar* or Tanganyika or Togo or Togolese or Uganda* or Zimbabw* or (Rhodesia* not "Rhodesian ridgeback") or Armenia* or Bhutan* or Bolivia* or Cameroon* or "Cape Verde" or "Cote d Ivoire" or "Ivory Coast" or Djibouti* or El next Salvador* or "Georgia Republic" or "Republic of Georgia" or Ghana* or Guatemala* or Guyana or Guyanese or Hondur* or Indonesia* or Kiribati or Kosovo* or Kosovar* or Laos or "Lao PDR" or LAO next People* or Laotian* or Lesotho* or Mauritania* or "Federated States of Micronesia" or Moldova* or Mongolia* or Morocco* or Nicaragua* or Nigeria* or Pakistan* or "Papua New Guinea" or Paraguay* or Philippines or (Filipino* or Filipina* not (Filipino next American* or "United States")) or (Samoa* not "American Samoa") or "Sao Tome" or Senegal* or Solomon next Island* or Sri next Lanka* or Ceylon or Sudan* or Swaziland or Syria* or "Timor-Leste" or "East Timor" or Ukrain* or Uzbekistan* or Vanuatu* or (Vietnam* not veteran*) or "West Bank" or "Gaza" or Palestin* or Yemen* or Zambia* or developing next countr* or LMIC or LMICs or "low income countries" or "low income country" or "low and middle income countries" or "low and middle income country" or "lower middle income countries" or "lower middle income country" or developing next nation* or developing next world or "developing economy" or "developing economies" or transitional next countr* or "global burden" or "global health" or global next orthop* or "global outreach" or "global public health" or global next watch or "international health" or "international public health" "world health" or "resource poor" or austere environment* or "third world" or central next Africa* or east next Africa* or eastern next Africa* or north next Africa* or northern next Africa* or southern next Africa* or west next africa* or western next africa* or sahara* or subsahara* or central next asia* or south next asia* or south next asia* or southeast next asia* or southeastern next asia* or southern next asia* or west next asia* or western next asia* or central next America* or eastern next Europe* or South next America* Publication Date from 2004 to 2014 (Word variations have been searched). 